Methods A cohort of HIV positive patients who had initially tested non-reactive to the TST were followed up for the period between November 2007 and February 2010. The Kaplan-Meier method was used to estimate the probability of not repeating the TST and Cox's Regression analysis used to analyse the factors associated with the time until TST was repeated. Cox's multivariate analysis was stratified according to the hospital the patients were followed, as this variable did not respect the principle of proportionality of risk. Results The probability of not repeating the TT by the end of the follow-up period was $42 \%$. The variables that remained associated with repetition of the TT in the final Cox multivariate model were: aged 40 years or more, BMI between 18.0 and 24.9, being female, and years of schooling.

Conclusion The analysis identified groups of individuals who should be the target of interventions aiming to ensure the TT is repeated following an initial non-reactive test in individuals infected with HIV.

\section{P2-496 AN INTERACTION OF SOCIAL SUPPORT AND REMOTENESS IN THE PREDICTION OF PSYCHOLOGICAL DISTRESS}

\section{doi:10.1136/jech.2011.142976m.23}

J Allen, ${ }^{*}$ K Inder, B Kelly, J Attia, T Lewin. Centre for Brain and Mental Health Research, University of Newcastle, Newcastle, New South Wales, Australia

Introduction The influence of social support on mental health outcomes may vary with remoteness in Australian communities. However, the potential variation of determinants of mental health with remoteness has rarely been directly examined. The current research aims to examine the association of social support and distress with remoteness.

Methods Community cohorts from rural and urban New South Wales were combined ( $N=5924$; mean age $=61.1 \mathrm{yrs}$; $44.2 \%$ Male). Standardised scores were used to facilitate assessment of participant satisfaction with social support networks as measured by the Duke Social Support Index and Interview Schedule for Social Interaction. Remoteness was assessed using a continuous measure, the Accessibility and Remote Index of Australia. The association between demographic characteristics, social support, remoteness, the interaction of social support and remoteness, and psychological distress (cut-off $>24$ on the Kessler 10) was studied using logistic regression. Results Younger age (OR 0.99; 95\% CI 0.98 to 0.99), not being in a married or defacto relationship (OR 1.50; 95\% CI 1.19 to 1.93 ) and decreased social support (OR $0.48 ; 95 \%$ CI 0.45 to 0.53 ), but not gender or remoteness, significantly predicted psychological distress. There was a significant interaction of social support by remoteness (OR 1.12; 95\% CI 1.03 to 1.22 ).

Conclusion Social support displayed a differential effect on distress outcomes across urban and rural environs. These results suggest that remoteness and associated community characteristics may moderate the protective influence of social support and that urban environments may be particularly influenced by very low levels of social support.

\section{P2-497 SELF-REPORT OF ORAL HEALTH IN THE ELDERLY AND ASSOCIATED FACTORS}

doi:10.1136/jech.2011.142976m.24

${ }^{1} \mathrm{D} D$ da Silva, ${ }^{2} \mathrm{R}$ B de Held, ${ }^{2} \mathrm{~S}$ V de Souza Torres, ${ }^{2} \mathrm{M} D \mathrm{D}$ R de Sousa, ${ }^{2} \mathrm{~A} L \mathrm{~L}$ Neri, ${ }^{3} \mathrm{~J}$ L F Antunes. ${ }^{1}$ Faculdade de Odontologia, Universidade de Sao Paulo, Sao Paulo, Sao Paulo, Brazil, '2Universidade Estadual de Campinas, Sao Paulo, Sao Paulo, Brazil; ${ }^{3}$ Faculdade de Saude Publica, Universidade de Sao Paulo, Sao Paulo, Sao Paulo, Brazil

Background The self-report of oral health is an index of subjective functional, social and emotional well-being related to oral status, and is an important determinant of the demand for dental services. This study aimed to assess this outcome and associated factors among the elderly.

Methods A cross-sectional study was conducted with 876 participants in a representative sample of elders (65 years or older) in Campinas, São Paulo, Brazil. Dental examinations followed criteria standardised by the WHO for epidemiological surveys on oral health. The self-report of oral health used the GOHAI-Geriatric Oral Health Assessment Index. Individuals were classified by sociodemographic characteristics, dental status and the prevalence of biological frailty. Data analysis used Poisson regression models, considering sample weights and the complex structure of cluster sampling.

Results Among participants, $18.3 \%$ preserved more than 20 teeth; $38.2 \%$ wore full dentures in both arches, $8.5 \%$ needed a denture in at least one dental arch. The average GOHAI index was high: 33.9 (maximum=36.0). Preserving more than 20 teeth, using denture in both arches, not needing such treatment, presenting with no alteration of oral mucosa and no signs of biological frailty were significantly associated with better self-perceived oral health $(\mathrm{p}<0.05)$.

Conclusions The assessment of self-perceived oral health allowed identifying the main factors that associate with this outcome. This tool may contribute to the planning of dental services, instructing strategies for health promotion aimed at an improved quality of life of people within this age group.

\section{P2-498 PARENTS' PERCEPTIONS AND PRACTICES REGARDING CONSANGUINITY RELATED TO $\beta$ - THALASSAEMIA: A MATCHED CASE CONTROL STUDY}

doi:10.1136/jech.2011.142976m.25

M Beg,* R Siddiqui, K Ahmad, K Humayun. Aga Khan University Hospital, Karachi, Pakistan

Introduction $\beta$-thalassaemia is most common genetically transmitted disorder in Eastern Mediterranean region including Pakistan. Like other recessive disorders, prevalence increases through consanguinity; Pakistan Demographic and Health Survey reports $50 \%$ consanguineous marriages. Perceptions about consanguinity in our population have recently not been studied. Objectives were to determine consanguinity related characteristics among parents of children with thalassaemia and without thalassaemia visiting a major health centre in Karachi-Pakistan. To compare consanguinity practices among such families and to assess susceptibility, benefits and barriers towards thalassaemia.

Methods Sample of 340 cases and 340 controls; age-sex matched were selected from February to April 2010. Parents of thalassaemic children and parents of non-thalassaemic children were interviewed after consent. Consanguinity related characteristics followed in families were inquired. Perceptions regarding susceptibility to thalassaemia, benefits and barriers to screening were determined. Conditional logistic regression used to calculate matched OR. Parents' approaches towards index child were assessed.

Results Compared to no cousin marriage, parents of thalassaemic children had more cousin marriages (MOR: $7.3,95 \%$ CI 3.6 to 14.6), and grand-parents cousin marriage (MOR: $2.8,95 \%$ CI 1.8 to 4.4 ) than controls, more cousin marriages occurred in immediate family in last 5 years of cases than controls (MOR: 2.7, 95\% CI 1.7 to 4.3 ) adjusting for ethnicity defined by mother tongue. Among case parents, (51\%) were taking index child to social events and only $21 \%$ were satisfied with attitudes of other's towards index child.

Conclusion Study supports hypothesis that among parents of thalassaemic (cases), practices and perceptions favouring consanguinity were greater as compared to parents of non-thalassaemic children (controls). 